\title{
RESEARCH
}

Open Access

\section{DNA topoisomerase II $\beta$ stimulates neurite outgrowth in neural differentiated human mesenchymal stem cells through regulation of Rho-GTPases (RhoA/Rock2 pathway) and Nurr1 expression}

Merve Zaim and Sevim Isik

\begin{abstract}
Background: DNA topoisomerase $\| \beta$ (topo $\| \beta$ ) is known to regulate neural differentiation by inducing the neuronal genes responsible for critical neural differentiation events such as neurite outgrowth and axon guidance. However, the pathways of axon growth controlled by topo $\| \beta$ have not been clarified yet. Microarray results of our previous study have shown that topo $\| \beta$ silencing in neural differentiated primary human mesenchymal stem cells (hMSCs) significantly alters the expression pattern of genes involved in neural polarity, axonal growth, and guidance, including Rho-GTPases. This study aims to further analyze the regulatory role of topo $\| \beta$ on the process of axon growth via regulation of Rho-GTPases.

Methods and results: For this purpose, topo $\| \beta$ was silenced in neurally differentiated hMSCs. Cells lost their morphology because of topo $\| \beta$ deficiency, becoming enlarged and flattened. Additionally, a reduction in both neural differentiation efficiency and neurite length, upregulation in RhoA and Rock2, downregulation in Cdc42 gene expression were detected. On the other hand, cells were transfected with topo $\| \beta$ gene to elucidate the possible neuroprotective effect of topo $\| \beta$ overexpression on neural-induced hMSCs. Topo $\| \beta$ overexpression prompted all the cells to exhibit neural cell morphology as characterized by longer neurites. RhoA and Rock2 expressions were downregulated, whereas Cdc42 expression was upregulated. Nurr1 expression level correlated with topo $\| \beta$ in both topo $\| \beta$-overexpressed and -silenced cells. Furthermore, differential translocation of Rho-GTPases was detected by immunostaining in response to topo $\| \beta$.

Conclusion: Our results suggest that topo $\| \beta$ deficiency could give rise to neurodegeneration through dysregulation of Rho-GTPases. However, further in-vivo research is needed to demonstrate if re-regulation of Rho GTPases by topo $\| \beta$ overexpression could be a neuroprotective treatment in the case of neurodegenerative diseases.
\end{abstract}

Keywords: Human mesenchymal stem cells, Neural differentiation, Neurite outgrowth, DNA topoisomerase II , Rho GTPases, Neurodegeneration

\footnotetext{
* Correspondence: sebimusan@gmail.com

SANKARA Brain and Biotechnology Research Center, Entertech Technocity,

Avcilar, 34320 Istanbul, Turkey
}

(c) The Author(s). 2018 Open Access This article is distributed under the terms of the Creative Commons Attribution 4.0 International License (http://creativecommons.org/licenses/by/4.0/), which permits unrestricted use, distribution, and reproduction in any medium, provided you give appropriate credit to the original author(s) and the source, provide a link to the Creative Commons license, and indicate if changes were made. The Creative Commons Public Domain Dedication waiver (http://creativecommons.org/publicdomain/zero/1.0/) applies to the data made available in this article, unless otherwise stated. 


\section{Background}

Type II DNA topoisomerases (topo II) are involved in double-strand cleavage and rejoining of nuclear DNA. In mammalian cells, there are two isoforms of topo II: topo II $\alpha$ and topo II $\beta[1,2]$. Unlike topo II $\alpha$, which is closely correlated with chromosomal segregation in dividing and pluripotent cells, topo II $\beta$ is involved in more specific processes and is intensively expressed in postmitotic and differentiated tissues $[3,4]$.

Studies have demonstrated that topo II $\beta$ has a significant role in neural differentiation during brain development by potentiating inducible neuronal genes to become transcribable [5-9]. Topo II $\beta$ inhibition in cultured cerebellar granule neurons (CGNs), dorsal root ganglions (DRGs), cortical neurons (CNs), and PC12 cells undergoing nerve growth factor (NGF)-induced differentiation significantly blocked neurite outgrowth and growth cone formation. Failure of neurons from topo II $\beta$ knockout mice to contact with muscle cells in cocultures could be due to the lack of topo II $\beta$-mediated neurite outgrowth [10]. In agreement with previous studies, we showed a decrease in neurite length due to topo II $\beta$ silencing of neural differentiated human mesenchymal stem cells (hMSCs) [11]. Topo II $\beta$ may be involved in the regulation of certain gene expressions required for neurite outgrowth during neural differentiation $[10,12,13]$.

Neurite outgrowth and growth cone formation are substantial steps in neuronal development [14]. Rho-GTPase family members (RhoA, Cdc42, Rac1), which are major intracellular regulators of neuronal polarity, integrate signals generated from extracellular matrix and reorganize the actin cytoskeleton, thereby arranging the morphology of neurites and growth cones [10, 15].

RhoA, a negative regulator of neurite outgrowth, induces growth cone collapse and neurite retraction. Rock2 is the major downstream target of RhoA in neural cells, and activation of RhoA results in axon growth inhibition through the RhoA/Rock2 pathway $[16,17]$. Unlike RhoA, Rac1 and Cdc42 give rise to an increase in growth cone development and neurite outgrowth [18, 19]. Moreover, these small GTPases regulate neuronal survival and death. Similar to their opposing functions in neurite outgrowth and growth cone formation, Cdc42/Rac1 activation promotes neuronal survival while Rho activation often elicits neuronal death $[18,19]$. Furthermore, Rho-GTPases play crucial roles in mediating nervous system development and neuronal survival and, thus, dysregulation of these GTPases lies behind the pathology of several neurodegenerative diseases including Alzheimer's disease (AD), Parkinson's disease (PD), Huntington's disease (HD), and amyotrophic lateral sclerosis (ALS) $[17,19]$.

In a previous study of ours, we investigated the target genes regulated by topo II $\beta$ in neural differentiation of hMSCs. According to the microarray results, topo II $\beta$ silencing appears to be important in several signal transduction pathways that contribute to neuronal polarization and pathogenesis of neurodegenerative diseases. In particular, genes involved in the regulation of neuronal cell morphology and cytoskeletal organization (e.g., Cofilin1, SORBS2) were found to be regulated by topo II $\beta$ [11]. Microarray analysis revealed that RND1 and RND2, atypical Rho members that are considered constitutively active and that antagonize the action of RhoA in the cytoskeletal organization by inducing neurite extensions, were downregulated in response to topo II $\beta$ silencing [11]. For further analysis, this study aims to evaluate the possible regulatory role of topo II $\beta$ on Rho-GTPases in axonal growth of neural differentiated hMSCs.

Neural differentiated MSCs are excellent tools and models for the treatment of nervous system disorders [20-23]. Although previous studies have shown that topo II $\beta$ promotes axonal growth in primary neurons [10], topo II $\beta$-related studies performed on hMSC-derived neural cells are very limited. Parenthetically, there have been no detailed report elucidating the downstream pathway of topo II $\beta$ in the regulation of neurite outgrowth.

In the present study, we discriminated the genes regulated by topo II $\beta$ in differentiation and neurite outgrowth by differentiating hMSCs into neural cells. Initially, we silenced topo II $\beta$ expression with specific small interfering (si)RNAs and examined how morphology, neural differentiation potential, axon growth, and Rho-GTPase expressions are affected by topo II $\beta$ deficiency. Then, we investigated the possible neuroprotective effect of topo II $\beta$ overexpression on neural differentiated hMSCs and elucidated the further involvement of Rho-GTPases in topo II $\beta$-mediated axon growth.

\section{Methods}

In this study, the hMSC line was assembled into six individual groups according to neural differentiation, topo II $\beta$ silencing, and overexpression (Table 1).

\section{Culture of bone marrow-derived hMSC line}

The bone marrow-derived hMSC (BM-hMSC) line (UE7T13 cells, no. RBRC-RCB2161), infected with retroviruses expressing papillomavirus E7 and hTERT to extend the lifespan of the cells [24-28], was purchased from Riken Science Institute, Japan. Cells were detached using 0.25\% Trypsin/EDTA (Gibco) solution when culture reached 80$90 \%$ confluency. The BM-hMSC line was seeded at a density of $3 \times 10^{3}$ cells $/ \mathrm{cm}^{2}$ in expansion medium (Dulbecco's modified Eagle's medium low glucose (DMEM-LG), 10\% fetal bovine serum (FBS), $0.1 \mathrm{mg} / \mathrm{ml}$ primocin) and incubated at $37{ }^{\circ} \mathrm{C}$, in a $5 \% \mathrm{CO}_{2}$ incubator. Subculture was repeated every 4 days. 
Table 1 Experimental groups and abbreviations used in the study

\begin{tabular}{ll}
\hline Abbreviations & Experimental groups \\
\hline hMSC & Control (untreated) human mesenchymal stem cells (hMSCs) \\
hMSC_mN3 & Neural differentiated hMSCs \\
hMSC_topo $\| \beta(-)$ & Topo $\| \beta$ transfected (silenced) hMSCs with siRNAs \\
hMSC_topo $\| \beta(+)$ & Topo $\| \beta$ transfected (overexpressing) hMSCs with pEGFP_topo $\| \beta$ \\
hMSC_topo $\| \beta(-) \_m N 3$ & Topo $\| \beta$ transfected (silenced) hMSCs with siRNAs induced to neural differentiation \\
hMSC_topo $\| \beta(+) \_m N 3$ & Topo $\| \beta$ transfected (overexpressing) hMSCs with pEGFP_topo $\| \beta$ induced to neural differentiation
\end{tabular}

\section{Immunophenotyping of hMSCs}

The hMSC line was analyzed by flow cytometry for the expression of hMSC specific cell surface antigens. Commonly used antibodies-CD45 (FITC), HLA-DR (PerCP) , CD34 (PE), CD73 (APC), CD90 (FITC), and CD105 (PE)-were used to characterize hMSC populations.

\section{Mesodermal differentiation of hMSCs Adipogenic differentiation}

For adipogenic differentiation, the hMSC line was harvested at passage 3 (p3) and seeded into 24-well plates at a density of $5 \times 10^{3}$ cells $/ \mathrm{cm}^{2}$. Cells were treated with complete MesenCult adipogenic medium containing MesenCult MSC basal medium (Stemcell) and 10\% adipogenic stimulatory supplement (Stemcell) for 21 days; adipogenic differentiation was confirmed by Oil Red O staining.

\section{Chondrogenic differentiation}

For chondrogenic differentiation, the hMSC line was harvested at p3 and seeded into 24-well plates at a density of 7 . $5 \times 10^{5}$ cells $/ \mathrm{cm}^{2}$. To stimulate chondrogenic differentiation, the culture medium was replaced with Stempro chondrocyte differentiation basal medium (Gibco) containing 10\% Stempro chondrogenesis supplement (Gibco). After 21 days of cultivation, the chondrogenic pellet was stained with Alcian Blue.

\section{Osteogenic differentiation}

For osteogenic differentiation, the hMSC line was seeded into 24-well plates at a density of $2 \times 10^{5}$ cells $/ \mathrm{cm}^{2}$. Osteogenic differentiation was stimulated by refreshing the expansion medium with complete MesenCult osteogenic medium including MesenCult MSC basal medium, osteogenic stimulatory supplement, dexamethasone, and ascorbic acid (all from Stemcell). After 5 days, when multilayering had been observed, $\beta$-glycerophosphate was added to complete MesenCult osteogenic medium. The chondrogenic pellet was assessed with Toluidine Blue staining after 5 weeks of cultivation.

\section{Neural differentiation of hMSCs}

hMSCs were seeded in culture medium at a density of $5 \times 10^{3}$ cells $/ \mathrm{cm}^{2}$ prior to neural induction. The induction medium was composed of several cytokines and growth factors, including $0.5 \mathrm{mg} / \mathrm{ml}$ dibutyryl cyclic AMP (dbcAMP; Sigma), $0.5 \mathrm{mM}$ 3-isobutyl-1-methylxanthine (IBMX; Sigma), $20 \mathrm{ng} / \mathrm{ml}$ human epidermal growth factor (hEGF; Sigma), $40 \mathrm{ng} / \mathrm{ml}$ recombinant human fibroblast growth factor (rhFGF; R\&D systems), $10 \mathrm{ng} / \mathrm{ml}$ fibroblast growth factor (FGF-8; Pepro Tech), $10 \mathrm{ng} / \mathrm{ml}$ recombinant human brain-derived neurotrophic factor (rhBDNF; R\&D systems), $2 \mathrm{mM}$ L-glutamine (Gibco), and $40 \mathrm{ng} / \mathrm{ml} \mathrm{NGF}$ in neurobasal medium (Gibco) supplemented with $2 \%$ B27 supplement (Gibco), and defined as modified N3 medium (mN3) as previously described [11]. Culture medium was changed with $\mathrm{mN} 3$ medium to induce neural differentiation. Cells in hMSC_topo II $\beta(-) \_\mathrm{mN} 3$ and hMSC_topo II $\beta(+) \_\mathrm{mN} 3$ groups were induced to neural differentiation $48 \mathrm{~h}$ post-transfection. $\mathrm{mN} 3$ medium was refreshed every $48 \mathrm{~h}$ for 5 days. Morphology of the cells was observed under an inverted phase contrast microscope.

\section{Immunostaining}

The hMSC line was permeabilized with TZN buffer (10 mM pH 7.5 Tris- $\mathrm{HCl}, 0.5 \%$ Nonidet P-40, $0.2 \mathrm{mM}$ $\mathrm{ZnCl}_{2}$ ) and fixed with $4 \%$ paraformaldehyde (PFA)/phosphate-buffered saline (PBS). After blocking with 10\% normal goat serum (NGS; Gibco) and 10\% normal horse serum (NHS; Biochrom) in 0.3\% PBS/Triton X (PBS-Tx), cells were treated with specific primary antibodies in PBS-Tx with 3\% NHS. Antibodies against neurofilament (NF; 1:100, Millipore), microtubule-associated protein (MAP2; 1:100, Promega), Tau (1:100, Santa Cruz), RhoA (1:100, Santa Cruz), Rac1 (1:100, BD), and Cdc42 (1:100, Santa Cruz) were used at the indicated dilutions. Following the washing steps, cells were treated with the secondary antibodies GAM-IgG-Alexa Fluor 488 (1:100, Invitrogen) and GAR-IgG-Alexa Fluor 594 (1:100, Invitrogen). Cells were then treated with 1:15,000× DAPI (Sigma) and, after final washings with PBS and distilled water, the slides were observed under a fluorescent microscope (Carl Zeiss).

\section{Silencing of topo II $\beta$ in hMSCs by siRNAs Determination of silencing efficiency}

Lipofectamine RNAiMAX reagent and four different validated topo II $\beta$-specific siRNAs were used to silence topo 
II $\beta$ expression at the mRNA level. The hMSC line was seeded at a density of $6 \times 10^{3}$ cells $/ \mathrm{cm}^{2}$. Briefly, siRNAs (7) $5,10,12.5,15$, and $20 \mathrm{nM}$ ) and reagent were diluted with Optimem, and siRNA-Lipofectamine RNAiMAX complexes were added to the cells. At $48 \mathrm{~h}$ post-transfection, total RNA of the topo II $\beta$-silenced hMSC line was extracted and efficiency was checked by real-time quantitative polymerase chain reaction (RT-qPCR). siRNA transfection was repeated every $48 \mathrm{~h}$ for 5 days for the hMSC_ topo II $\beta(-)$ and hMSC_topo II $\beta(-) \_\mathrm{mN} 3$ groups.

\section{Cell viability}

To determine the optimum concentration of topo II $\beta$ specific siRNAs on the hMSC line, an MTT cell proliferation assay (Roche) was performed. The hMSC line was seeded in 96-well plates at a density of $2 \times 10^{3}$ cells /well. Four different validated siRNAs (topo II $\beta-5$, topo II $\beta-6$, topo II $\beta-7$, and topo II $\beta-8$; Qiagen) and Lipofectamine RNAiMAX reagent were used for silencing topo II $\beta$. siRNAs $(7.5,10,12.5,15$, and $20 \mathrm{nM})$ and reagent were diluted with Optimem and incubated for $15 \mathrm{~min}$ at room temperature to allow siRNA-Lipofectamine RNAiMAX complex formation. siRNA-Lipofectamine RNAiMAX complexes were added to the wells. After $48 \mathrm{~h}$ of siRNA transfection, a cell proliferation assay was performed with the MTT reagent (Roche) according to the manufacturer's instructions. Absorbance was measured at $490 \mathrm{~nm}$ using a microplate reader (Synergy HT; Biotek).

\section{Transfection of hMSCs with topo $\| \beta$ gene Topo $\| \beta$ plasmid isolation and purification}

The pEGFP-N1 plasmid including topo II $\beta$ gene was kindly gifted by Prof. Tsutsui from Okayama University Medical School. The EGFP-N1 plasmid was transformed to calcium shocked competent E. coli $\mathrm{DH} 5 \alpha$ strain for amplification and plasmid isolation was performed with the Plasmid Isolation Kit (Qiagen) according to the manufacturer's instructions. Before transfection, confirmation of topo II $\beta$ gene insert was carried out by digestion of the plasmid with appropriate restriction enzymes (XhoI, SmaI, and BamHI) and colony PCR.

\section{Determination of overexpression efficiency}

To transfect the hMSC line with topo II $\beta$ gene, 4D Nucleofector $^{\text {rat }}$ system (Lonza) was used; $5 \times 10^{5}$ cells of the hMSC line were transfected with five different concentrations of topo II $\beta$ plasmid $(4,5,6,8$, and $10 \mu \mathrm{g})$ using the $4 \mathrm{D}$ Nucleofector $^{\text {rw }}$ system (Lonza). hMSCs were detached by 0 . 25\% Trypsin/EDTA (Gibco) and resuspended in a total of $100 \mu \mathrm{l}$ of solution, including $82 \mu \mathrm{l}$ nucleofector solution and $18 \mu \mathrm{l}$ supplement. Topo II $\beta$ plasmid $(4,5,6,8$, and $10 \mu \mathrm{g}$ ) were added to each $100 \mu \mathrm{l}$ solution and transferred into nucleocuvettes, respectively. The FF-104 (high-efficiency) program was applied. After nucleofection, cells were resuspended in $500 \mu \mathrm{l}$ prewarmed RPMI containing 10\% FBS and incubated at $37^{\circ} \mathrm{C}$ for $10 \mathrm{~min}$ as a recovery step. The transfected hMSC line was seeded into culture dishes containing the DMEM-LG and 10\% FBS and incubated at $37{ }^{\circ} \mathrm{C}$ in a $5 \% \mathrm{CO}_{2}$ incubator. The medium was refreshed $24 \mathrm{~h}$ after nucleofection. Total RNA was extracted and the efficiency of overexpression was measured by RT-qPCR (Corbett Life Science).

\section{Cell viability}

To determine the plasmid concentration-based cytotoxicity, an MTT viability assay was performed. Briefly, the hMSC line was detached and resuspended in a total of $100 \mu \mathrm{l}$ of nucleofection solution including 4, 5, 6, 8, and $10 \mu \mathrm{g}$ of topo II $\beta$ plasmid and transferred into nucleocuvettes, respectively. After nucleofection and a recovery step, cells were seeded into culture dishes containing the DMEM-LG and $10 \% \mathrm{FBS}$ and incubated at $37{ }^{\circ} \mathrm{C}$ in a $5 \%$ $\mathrm{CO}_{2}$ incubator. After $48 \mathrm{~h}$ of topo II $\beta$ transfection, a cell proliferation assay was performed with the MTT reagent (Roche) according to the manufacturer's instructions. Absorbance was measured at $490 \mathrm{~nm}$ using a microplate reader (Synergy HT; Biotek).

\section{Reverse transcription quantitative PCR (RT-qPCR)}

Total RNA was extracted using the RNeasy kit (Qiagen) and reverse transcribed into cDNA by the Quantitect Reverse Transcription Kit (Qiagen) according to the manufacturer's instructions.

Real-time PCR amplification was carried out using cDNA samples, gene specific primers, $\mathrm{ddH}_{2} \mathrm{O}$, and the SYBR Premix Ex Taq (Tli RNase H Plus) including second generation dye SYBRGreen on a Rotor Gene 6000 Real Time PCR instrument (Corbett Life Science) under given thermocycling conditions. Expression levels of GAPDH, Topo II $\beta$, RhoA, Cdc42, Rac1, Rock2, and Nurr1 primers were determined using the $\Delta \Delta \mathrm{Ct}$ formula according to Pfaffl [29]. Primer sequences are indicated in Table 2. A standard dilution graph was drawn according to the GAPDH primer. $\mathrm{Ct}$ values of the remaining primers were normalized according to a standard dilution graph. Triplicate samples were used, and experiments were repeated three times.

\section{Neurite outgrowth}

Neurite length per neural cell was determined as the sum of the lengths of all neurites of a single neural cell, measured with NIS Elements software (Nikon) and calculated using the ImageJ software (NIH). The average total neurite length per group was determined from at least 100 images from random fields. Images were investigated not for only length measurements but also calculation of neural differentiation efficiency. 
Table 2 Primer sequences for reverse transcription quantitative polymerase chain reaction (RT-qPCR)

\begin{tabular}{llll}
\hline Primers & Forward & Reverse & $\begin{array}{c}\text { Annealing } \\
\text { Temperature }\end{array}$ \\
\hline GAPDH & GCGAGATCCCTCCAAAATCAA & GTTCACACCCATGACGAACAT & $60{ }^{\circ} \mathrm{C}$ \\
Topo $\| \beta$ & GGGCTTAGGGACTGTATCTGAA & $60{ }^{\circ} \mathrm{C}$ \\
RhoA & CTITCACCATCATTGGTCTG & GCGATCATAATCTTCCTGCC & $55^{\circ} \mathrm{C}$ \\
Rac1 & AACCAATGCATTCCTGGAG & CAGATTCACCGGTTTCCAT & $60^{\circ} \mathrm{C}$ \\
Cdc42 & CTCCGGAAACTCAACCCAAA & GACGCAGAGGCTTCAAACAG & $60^{\circ} \mathrm{C}$ \\
Rock2 & TTGCTCTGGATGCAATACACTC & TCTCGCCCATAGAAACCATCA & $55.5^{\circ} \mathrm{C}$ \\
Nurr1 & CTTGTGTTCAGGCGCAGTATG & GAGTGGTAACTGTAGCTCTGAGAAGC & $60{ }^{\circ} \mathrm{C}$ \\
\hline
\end{tabular}

\section{Statistical analyses}

Data are presented as mean \pm standard deviation (SD) of three independent experiments. The paired $t$ test was used to evaluate the differences between the experimental groups. Differences were considered statistically significant at ${ }^{*} p<0.01$ and ${ }^{* * *} p<0.001$.

\section{Results}

mN3 cytokine combination induced successful neural differentiation in hMSCs

To evaluate the neural differentiation of the BM-hMSC line, we initially demonstrated that our data were in agreement with the criteria of the International Society for Cellular Therapy. BM-hMSCs were adherent to plastic in culture with typical fibroblastic morphology, and cells maintained their morphology up to p30 under culture conditions.

For characterization of the hMSC line, the immunophenotypic cell surface profile for CD45, HLA-DR, CD105, CD34, CD73, and CD90 were analyzed by flow cytometry; cells were positive for the markers CD73, CD90, and CD105, and negative for CD45, CD34, and HLA-DR (Additional file 1: Figure S1).

The multilineage mesodermal differentiation potential of the hMSC line was assessed by differentiating cells into adipocytes, osteocytes, and chondrocytes by incubating under appropriate in-vitro conditions. Lipid vacuoles in adipocytes were observed 3 weeks after adipogenic induction, following staining with Oil Red O. After 5 weeks, hMSCs generated aggregates or nodules that were stained positive by Toluidine Blue under osteogenic culture conditions, and calcium accumulation was obtained. Chondrogenic cell pellets showed strong histological staining with Alcian Blue for the presence of proteoglycans (Additional file 2: Figure S2).

The hMSC line was induced to neural differentiation by treating cells with the $\mathrm{mN} 3$ cytokine combination for 5 days in culture. $\mathrm{mN} 3$, a variation of $\mathrm{N} 3$ neural induction cocktail first suggested by Long et al. [30], is a nontoxic cytokine combination with high neural differentiation efficiency. Throughout the differentiation process, cells were seen to structurally adopt a polarized phenotype with longer neurites generating a neuronal network within $24 \mathrm{~h}$ while they were still in the process of neural maturation (Fig. 1A). To confirm neural differentiation of the hMSC line, immunofluorescent staining was performed 5 days after induction. Neural differentiated hMSCs stained positive for the neural markers NF (98\%), Tau (99\%), and MAP2 (100\%) (Fig. 1B). No glial marker expression (glial fibrillary acidic protein (GFAP)) was seen. Taken together, the results demonstrate that $\mathrm{mN} 3$ induced cell morphology changes consistent with neural differentiation, and this was validated by increased expression of known neural differentiation markers. These findings demonstrate that $\mathrm{mN} 3$ treatment enhances differentiation of the hMSC line in vitro.

\section{Topo II $\beta$ expression is crucial for neural differentiation and neurite outgrowth of neural-induced hMSCs}

Topo II $\beta$ expression was silenced using four different validated siRNAs targeting human topo II $\beta$ mRNA. To detect the optimum (>90\%) topo II $\beta$-specific siRNA concentration, $7.5,10,12.5,15$, and $20 \mathrm{nM}$ siRNAs was used. RTqPCR results of topo II $\beta$-silenced hMSCs showed that 7 . $5 \mathrm{nM}$ siRNA can silence topo II $\beta$ gene expression $(85 \pm 2 \%)$ $(p<0.01)$; however, maximal knockdown $(>95 \%) \quad(p<0$. 001) was obtained using $10 \mathrm{nM}$ siRNA, and increasing the siRNA concentration from $10 \mathrm{nM}$ to $20 \mathrm{nM}$ did not enhance the silencing efficiency (Fig. 2a).

A cell proliferation and cytotoxicity assay based on MTT was performed to elucidate the effects of different concentrations of siRNAs on proliferation of the hMSC line. A cell viability graph of untransfected and topo II $\beta$-silenced hMSCs revealed that viability of transfected cells was not affected by siRNAs compared with untransfected cells. Results indicated that siRNA concentrations from 7.5 to $20 \mathrm{nM}$ did not have a significant cytotoxic effect on cells (Fig. 2b). We obtained highly efficient siRNA transfection (>95\%) using $10 \mathrm{nM}$ siRNA without any significant cytotoxicity (Fig. 2b).

The plasmid pEGFP-topo II $\beta$, which encodes the fulllength rat topo II $\beta$ with enhanced green fluorescent protein (EGFP) sequence fused at the C-terminus, was constructed and kindly gifted by Prof. Tsutsui from Okayama University Medical School, Japan. Transfection of the hMSC line with different concentrations of topo II $\beta$ plasmid $(4,5,6,8$, and 

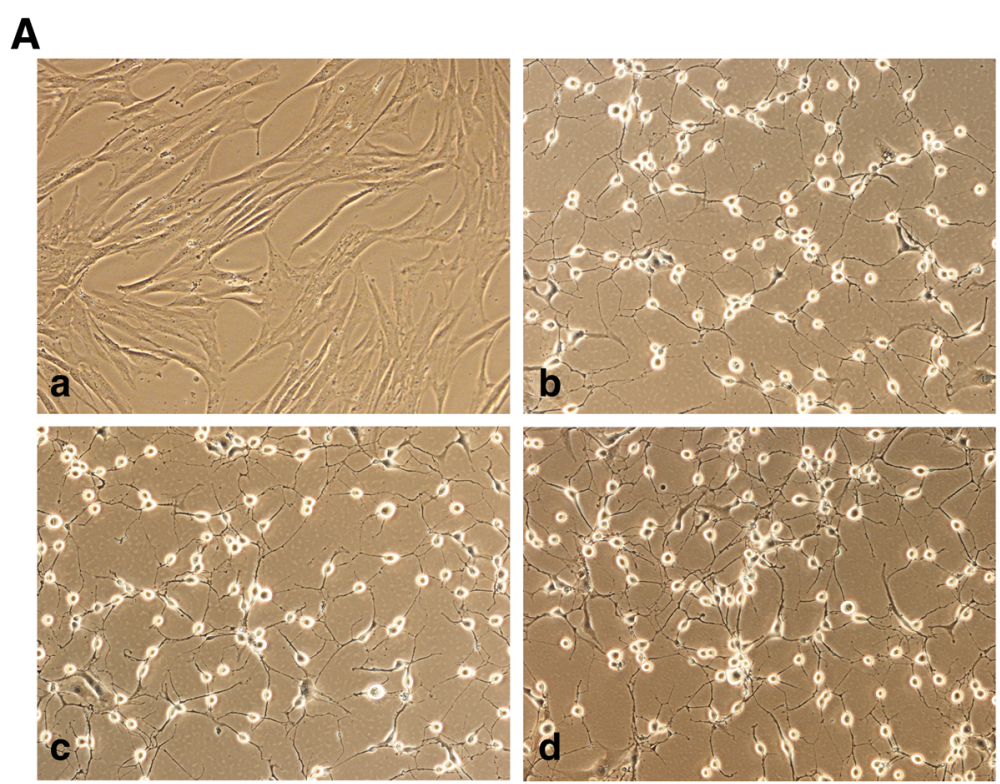

B

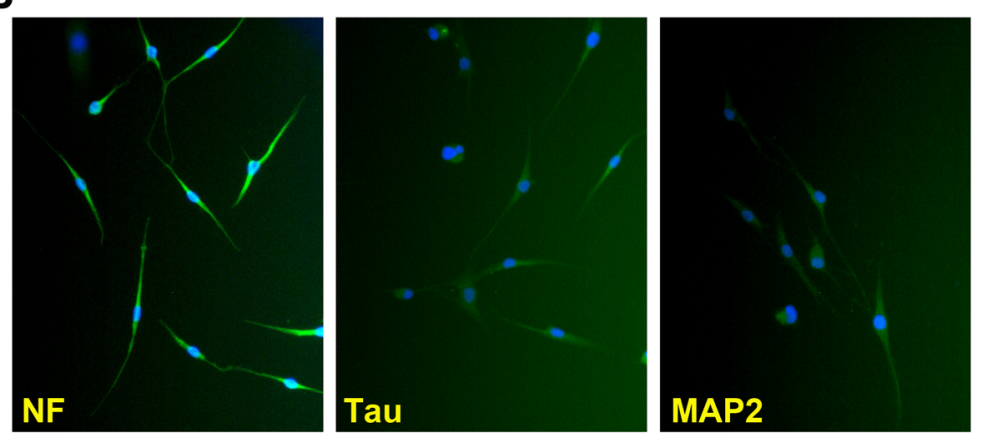

Fig. 1 Neural differentiation of hMSCs with the mN3 cytokine combination. A Undifferentiated hMSCs (control, a), neural differentiated hMSCs at day 1 (b), day 3 (c) and day 5 (d). mN3 treatment induced hMSC cellular differentiation characterized by a polar appearance with long, out-branching axons. B Immunostaining of neural differentiated hMSCs at day 5 with the neural markers neurofilament (NF), Tau, and microtubule-associated protein (MAP2). mN3 treatment induced the expression of neural differentiation markers (images $\times 10$ )

$10 \mu \mathrm{g})$ was performed using the FF-104 (high-efficiency) program of the 4D Nucleofector ${ }^{\text {Tw }}$ system (Lonza) to determine the optimum plasmid concentration with maximum transfection efficiency and minimum cytotoxicity. Transfection of hMSCs with 6 and $8 \mu \mathrm{g}$ plasmid resulted in the highest topo II $\beta$ gene expression level among other transfected cells (Fig. 2c).

The MTT cell proliferation assay was performed to determine specific toxicity of each topo II $\beta$ plasmid concentration $(4,5,6,8$, and $10 \mu \mathrm{g})$ on the hMSC line. According to the graph (Fig. 2d), increasing the plasmid concentration up to $6 \mu \mathrm{g}$ did not have any cytotoxic effect on transfected cells compared with control cells. Therefore, $6 \mu \mathrm{g}$ plasmid was used in further transfection experiments.

To identify whether topo II $\beta$ silencing or overexpression alters the cell morphology, hMSCs were transfected with siRNAs or pEGFP_topo II $\beta$ without neural induction. The untransfected (control) hMSC line had fibroblastic and spindle-shaped morphology, whereas topo II $\beta$-silenced hMSCs lost their characteristic morphology and became enlarged and flattened. Only GFP-transfected cells showed the same morphology as untransfected cells (data not shown). Topo II $\beta$ transfected hMSCs were fibroblastic and spindle-shaped as well and maintained their morphology after transfection, furthermore gaining longer and thinner cellular processes. Morphological changes were observed $24 \mathrm{~h}$ after transfection (Fig. 2e). RT-qPCR was performed to confirm overexpression and silencing of topo II $\beta$ in the hMSC line at the mRNA level. Figure $2 \mathrm{f}$ shows a 4.5 -fold increase $(p<0.01)$ in hMSC_topo II $\beta(+)$ and a 25 -fold $(p<0$. 001) suppression in hMSC_topo II $\beta(-)$ compared with untransfected cells. This result indicates a sufficient overexpression and knockdown of cells $48 \mathrm{~h}$ post-transfection.

To identify how topo II $\beta$ silencing or overexpression affects the neural differentiation potential and neurite lengths of neural-induced hMSCs, we assessed the 

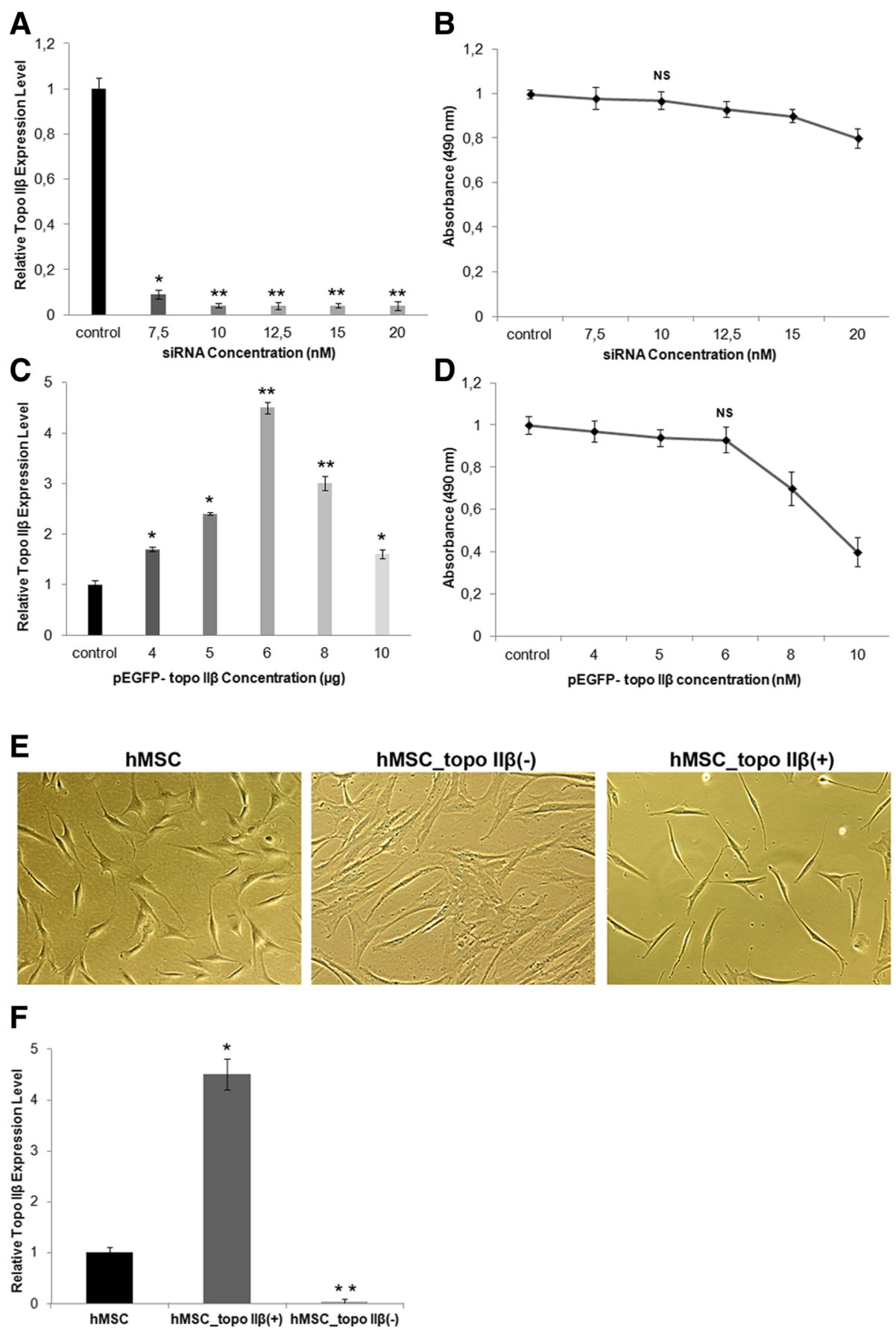

Fig. 2 Detection and quantification of topoisomerase (topo) $\| \beta$ overexpression and silencing in hMSCs. a Topo $\| \beta$ was silenced using 7.5, 10, $12.5,15$, and $20 \mathrm{nM}$ topo $\| \beta$-specific small interfering (si)RNAs and silencing efficiency was determined by RT-qPCR. $\mathbf{b}$ siRNA-based cytotoxicity for each siRNA concentration was evaluated by MTT cell proliferation assay. c Topo $\| \beta$ was overexpressed using 4, 5, 6, 8, and $10 \mu \mathrm{g}$ pEGFP-topo $\| \beta$ and transfection efficiency was quantified by RT-qPCR. $\mathbf{d}$ EGFP-topo $\| \beta$ plasmid-based cytotoxicity for each plasmid concentration was evaluated by MTT cell proliferation assay. e Morphology of control (untransfected hMSCs), topo II-silenced, and -overexpressed hMSCs. f Topo II expression levels of topo II-silenced (10 nm siRNA) and -overexpressed ( $6 \mu \mathrm{g}$ pEGFP-topo II $\beta$ ) hMSCs were compared by RT-qPCR. Images $\times 10$. Error bars represent the means \pm standard deviation; ${ }^{*} p<0.01,{ }^{* *} p<0.001$. NS, nonsignificant

differentiation potential and neurite lengths of each group over 5 days. Neural differentiation efficiencies and neurite lengths of untransfected, topo II $\beta$-silenced, and overexpressing hMSC line were compared at days 1,3 , and 5 (Fig. 3). Neural differentiation efficiency was quantified by determining the percentage of cells both 


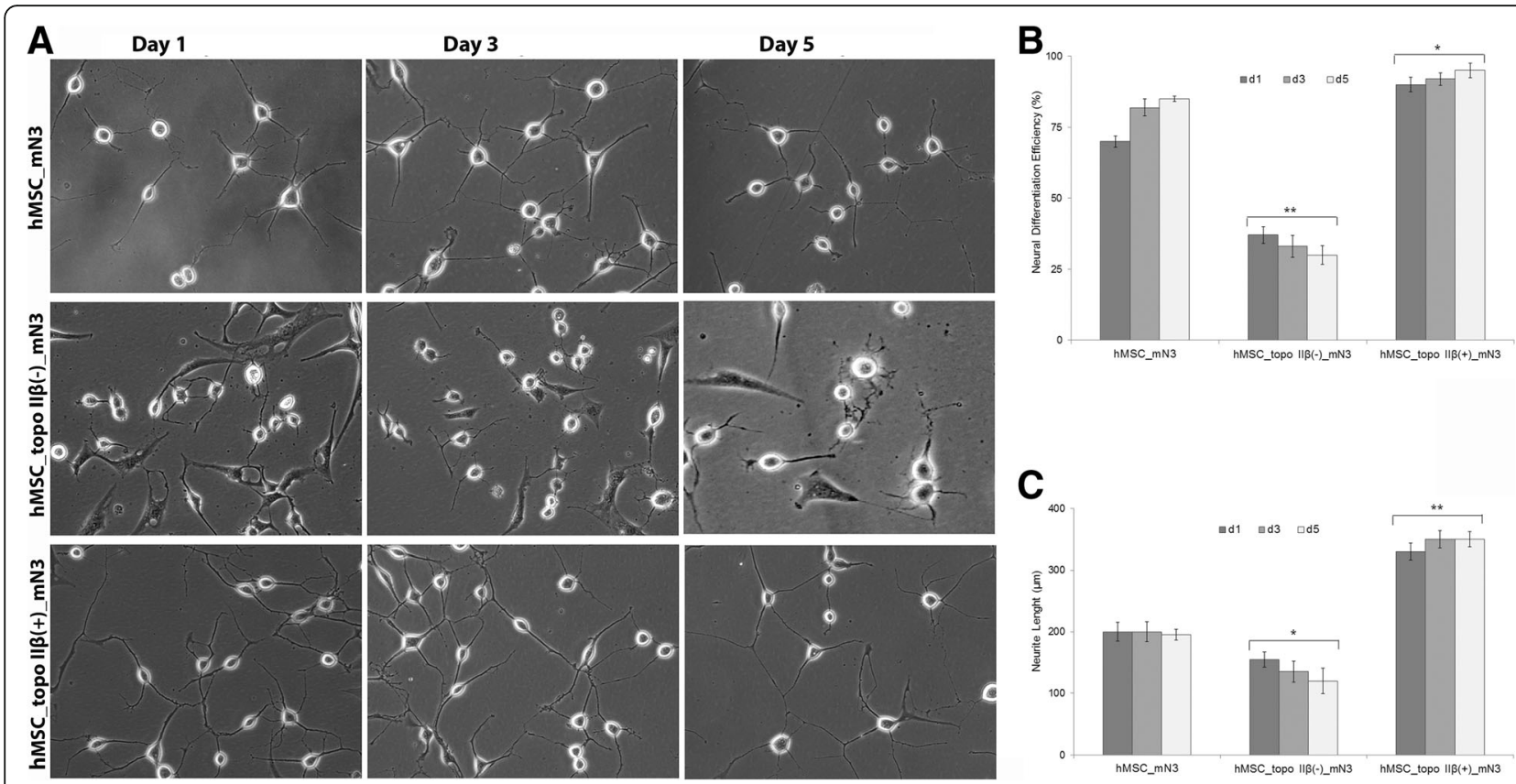

Fig. 3 Neural differentiation of untransfected, topo $\| \beta$-silenced, and -overexpressed hMSCs. a Morphology of untransfected, topo $\| \beta$-silenced, and -overexpressed hMSCs induced to neural differentiation by the mN3 cytokine combination for 5 days. b Neural differentiation efficiencies and $\mathbf{c}$ neurite lengths of topo $\| \beta$-silenced and -overexpressed cells at day (d)1, day 3, and day 5 were compared with untransfected cells. Both neural differentiation efficiency and neurite length were directly proportional to topo $\| \beta$ expression. The average total neurite length and neural differentiation efficiency per group for each day was determined from at least 100 images from random fields.

Images $\times 10$. Error bars represent the means \pm standard deviation; ${ }^{*} p<0.01,{ }^{* *} p<0.001$

positive for neural markers and having neural-like morphology. As shown in Fig. 3b, untransfected cells exhibited high levels of neural differentiation potential starting from $70 \pm 2 \%$ at day 1 and reached $85 \pm 5$ at day 5 . The neurogenic potential of topo II $\beta$-deficient cells was extremely low ( $37 \pm 3 \%$ at day 1$)$ compared with untransfected cells $(p<0.001)$ and decreased during the differentiation process $(30 \pm 4 \%$ at day 5$)$. On the other hand, transfection of the hMSC line with topo II $\beta$ resulted in an increased neural differentiation efficiency from $90 \pm 3 \%$ (day 1$)$ to $95 \pm 5 \%$ (day 5$)(p<0.01)$. Neurite lengths were measured using the NIS Elements software (Nikon) and ImageJ (NIH) program as described in the Methods section. Neurite outgrowth in topo II $\beta$ deficient cells decreased from $200 \pm 15 \%$ to $155 \pm 12 \%$ at day 1 , and $195 \pm 9 \%$ to $120 \pm 18 \%$ at day 5 compared with untransfected cells $(p<0.01)$. Measurements of the neurite lengths of topo II $\beta$-overexpressing cells showed increased neurite length, with $330 \pm 12 \%$ at day 1 and $350 \pm 8 \%$ at day $5(p<0.001)$ (Fig. 3c). Results indicate that topo II $\beta$ silencing decreased the neural differentiation potential of the hMSC line as most cells were unresponsive to differentiation and inhibited neurite outgrowth, whereas topo II $\beta$ overexpression increased the neural differentiation potential of $\mathrm{mN} 3$-induced hMSCs since almost all cells showed neuronal morphology and induced neurite outgrowth (Fig. 3).

\section{Topo $I \beta$ regulates axon growth and maintenance} through Rho-GTPases in neural differentiated hMSCs

Untransfected, topo II $\beta$-silenced, and overexpressed hMSCs were induced to neural differentiation with the $\mathrm{mN} 3$ cytokine combination, and cells were immunostained with Rho-GTPase (RhoA, Cdc42, Rac1) and neural marker (Tau, NF, MAP2) antibodies on the fifth day of neural induction (Fig. 4).

Immunofluorescence staining showed that all the neural differentiated hMSCs stained positively for the neural markers NF, Tau, and MAP2. In neural differentiated cells lacking topo II $\beta$ expression, the neural differentiation efficiency decreased, and nondifferentiated cells expanded morphologically and their nuclei grew in size. As a result of the diminished neural differentiation efficiency, the expression of the neural markers was reduced (the dominant color was red).

Rho-GTPase expressions were detected in both the cytoplasm and the nucleus, but their localizations were changed among groups. While colocalization of the neural marker and Rho-GTPase expressions were detected in untransfected neural cells (shown in yellow in Fig. 4a), Rac1 and especially Cdc42 immunoreactivity (shown in red in Fig. 4a) was enriched in the growing tips of prolonged axons in topo II $\beta$-overexpressing cells (Fig. 4a).

RhoA was found to be expressed in both the cytoplasm and the nucleus of untransfected cells. However, RhoA 

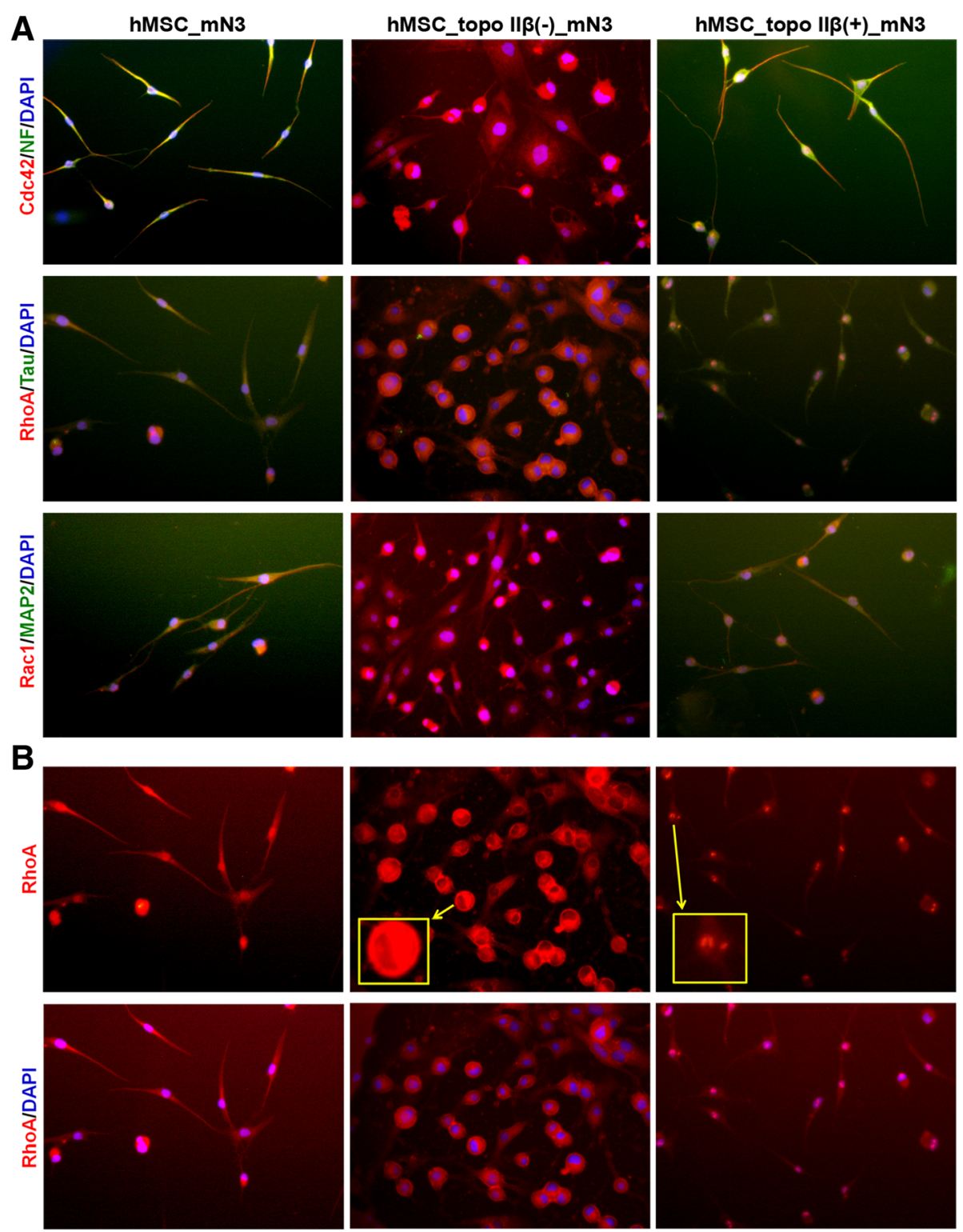

Fig. 4 Immunostaining of hMSC_mN3, hMSC_topo $\| \beta(-) \_m N 3$, and hMSC_topo $\| \beta(+) \_m N 3$ cells with the neural markers neurofilament (NF), Tau, microtubule-associated protein (MAP2), and Rho-GTPases Cdc42, RhoA, and Rac1. In hMSC_mN3 cells, the arrows indicate the overlapping of the neural marker NF and Rho-GTPase Cdc42 expressions (yellow). In hMSC_topo II $(+)$ _mN3 cells, the arrows indicate Rho-GTPase Cdc42 expression in extending the ends of axons (red) (a). Immunostaining of hMSC_mN3, hMSC_topo II(-)_mN3 and hMSC_topo II (+)_mN3 cells with RhoA. Arrows indicate the lack of RhoA expression in the nucleus of hMSC_topo $\| \beta(-) \_m N 3$ and the enhanced RhoA expression in the nucleus of hMSC_topo $\| \beta(+) \_m N 3$ cells $(\mathbf{b})$. Images $\times 10$, magnified nucleus $\times 20$

expression was not detected in the nucleus of topo II $\beta$ silenced cells. On the other hand, unlike topo II $\beta$-deficient cells, RhoA expression was enriched in the nuclear regions and was especially localized in the nucleolus in topo II $\beta$ overexpressing cells (Fig. 4b).

\section{Topo II $\beta$ regulates expression of Nurr1, Rho-GTPases} (Cdc42, RhoA, Rac1), and Rock2

Relative quantification was performed to calculate the RTqPCR results of Topo II $\beta$, Nurr1, Cdc42, RhoA, Rac1 and
Rock2 gene expressions (Fig. 5). A standard curve using the housekeeping gene GAPDH was used as an internal control and, according to the curve, RT-qPCR efficiency was over $99 \%$ which is within the accepted range of efficiency.

Cells in each experimental group were cultured for 5 days and mRNA levels of topo II $\beta$ gene in each group were determined at days 1,3 , and 5 by RT-qPCR. The topo II $\beta$ expression pattern of hMSC_mN3 cells gradually increased with the number of days in culture compared with 

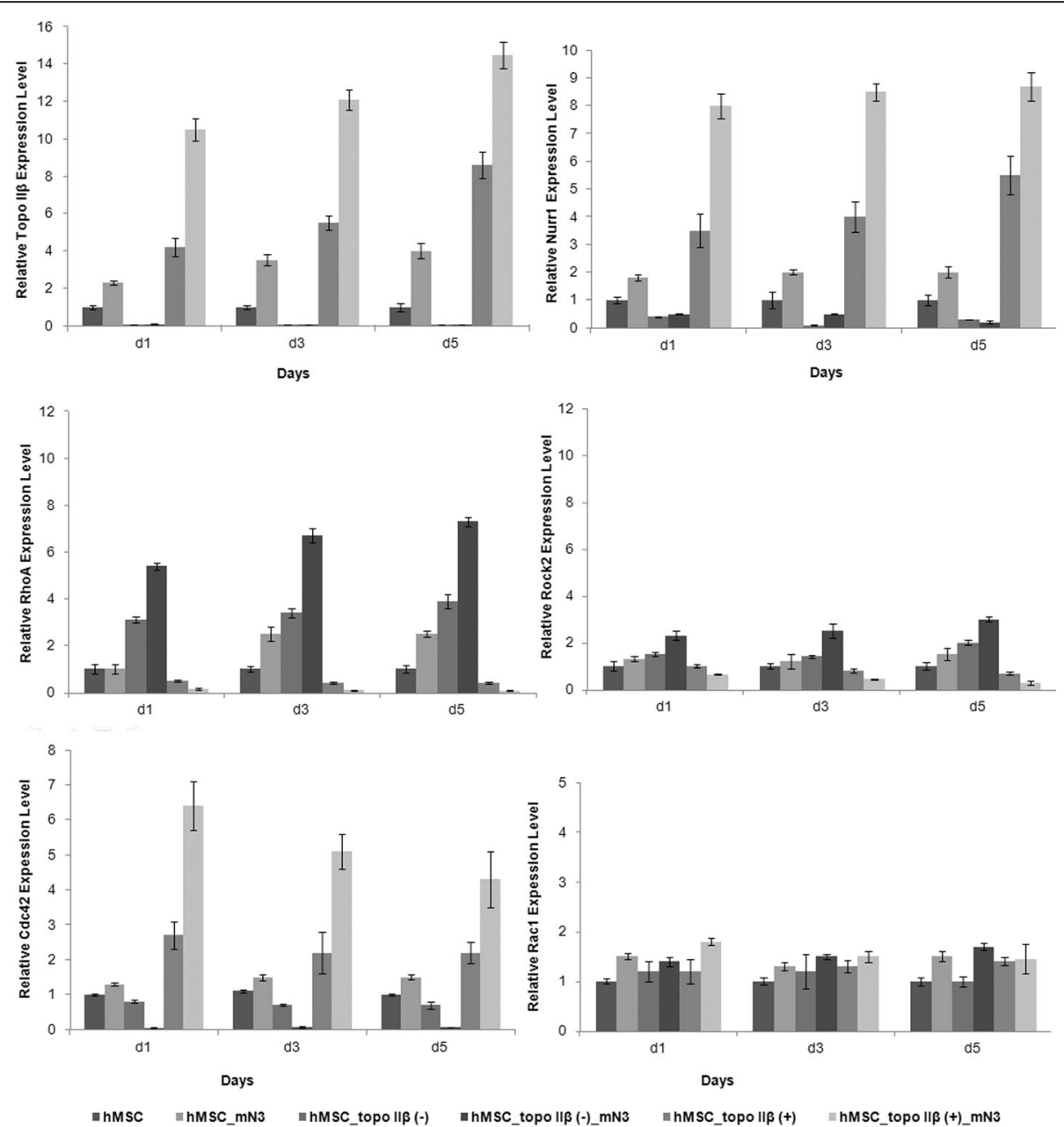

Fig. 5 RT-qPCR results of relative Topo $\| \beta$, Nurr1, Rho GTPase (RhoA, Rac1, and Cdc42), and Rock2 expressions at days (d)1, 3, and 5 for each group. Nurr1 showed nearly the same pattern as topo $\| \beta$, since the expression increased due to neural differentiation and topo $\| \beta$ transfection. Cdc42 also exhibited a similar expression pattern to topo $\| \beta$ and Nurr1, being upregulated due to neural induction and topo $\| \beta$ overexpression. RhoA and the downstream effector kinase Rock2 expressions were inversely correlated with topo $\| \beta$, Nurr 1 , and Cdc42 expressions. Neural induction and topo $\| \beta$ overexpression increased Nurr 1 and Cdc42 expression, whereas decreased RhoA and Rock2. Rac1 expression was independent. Error bars represent the means \pm standard deviation; $n=3$ samples in triplicate; ${ }^{*} p<0.01,{ }^{* *} p<0.001$

untransfected hMSCs. As a result of topo II $\beta$ knockdown, expression levels were suppressed in hMSC_topo II $\beta(-)$ and hMSC_topo II $\beta(-)$ mN3 cells $(p<0.001)$. hMSC_topo $\mathrm{II} \beta(+)$ cells exhibited higher topo II $\beta$ expression levels compared with untransfected cells $(p<0.001)$. The highest topo II $\beta$ levels were detected in hMSC_topo II $\beta(+) \_\mathrm{mN} 3$ cells and the expression pattern increased with days in culture $(p<0.001)$.

The expression level of RhoA, a well-known negative regulator of neural differentiation and neurite outgrowth, was increased in hMSC_topo II $\beta(-)(p<0.01)$ and hMSC topo II $\beta(-)$ mNN3 cells compared with untransfected cells $(p<0.001)$. On the other hand, a decreased RhoA expression level was detected in hMSC_topo II $\beta(+) \_\mathrm{mN} 3$ cells $(p<0.001)$, showing a negative correlation between RhoA and topo II $\beta$ gene expressions.
Cdc42, which promotes neurite outgrowth, was downregulated in response to topo II $\beta$ deficiency $(p<0.001)$, whereas the expression level was upregulated in topo II $\beta$ overexpressing neural cells $(p<0.01)$. Furthermore, topo $\mathrm{II} \beta$ overexpression in $\mathrm{mN} 3$-treated hMSCs induced an increase in the Cdc42 gene expression level at day $1(p<$ 0.001 ) which decreased gradually over the following days.

Except for heterogeneity in the expression levels between days, no significant difference was found in Rac1 expression between each group. In contrast to the known positive regulatory role of Rac1 in neurite outgrowth, RT-qPCR results showed no correlation with topo II $\beta$ deficiency or overexpression.

It has been shown that RhoA activates Rock2, major downstream target protein of RhoA in neural cells, and that the RhoA/Rock2 signaling pathway leads to axon 
growth inhibition [17]. Depending upon the increased RhoA expression level in topo II $\beta$-silenced cells, we also investigated the effect of topo II $\beta$ deficiency on Rock2 gene expression. As expected, the Rock 2 gene expression level was increased as a result of raised RhoA expression levels in response to topo II $\beta$ silencing. Accordingly, topo II $\beta$-transfected cells showed a reduction in the expression levels of Rock2 $(p<0.01)$.

Heng et al. demonstrated that topo II $\beta$ was a downstream target of Nurr1, and the expression of the enzyme was downregulated in Nurr1 knockout mice [13]. RT-qPCR analysis was performed to check the expression pattern of Nurr1 in topo II $\beta$ silenced and overexpressing hMSCs. The expression level of Nurr1 was both upregulated in hMSC_ topo II $\beta(+)$ and hMSC_topo II $\beta(+)$ mN3 cells $(p<0.001)$, whereas it was downregulated in hMSC_topo II $\beta(-)$ and hMSC_topo II $\beta(-) \_\mathrm{mN} 3$ cells $(p<0.01)$ compared with the control during the whole differentiation process. The Nurr1 expression level was highly correlated with topo II $\beta$ in response to topo II $\beta$ silencing and overexpression.

\section{Discussion}

Topo II $\beta$ activity is known to regulate neural differentiation by potentiating neuronal genes that are responsible for neural differentiation processes such as neurite outgrowth $[5,10]$. Additionally, preliminary microarray results proposed that topo II $\beta$ silencing appears to be important in several signal transduction pathways which may contribute to neuronal polarization and pathogenesis of neurodegenerative diseases [11]. On the other hand, Rho-GTPases regulate neuronal polarization, and dysregulation of these GTPases lies behind the pathology of neurodegeneration [19]. However, there have been no reports analyzing the link between topo II $\beta$ and Rho-GTPases. In this study, for the first time, we identify that topo $\mathrm{II} \beta$ has a regulatory role in the process of axonogenesis via regulation of RhoGTPases.

Since their neural transdifferentiation capability was first shown, primary hMSCs have been promising candidates for the treatment of damaged neural tissue. However, apart from the apparent ethical issues, the use of primary hMSCs has been limited due to their dependency on conditions such as passage number and donor-related heterogeneity. The low frequency of MSCs necessitates their in-vitro expansion prior to use, and primary cells can be provided for only a limited time before they undergo cellular senescence [31-33]. To obtain sufficient numbers of a homogenous hMSC population and exclude inter-donor variability, the clonally immortalized hMSC line was chosen to make an in-vitro human neural differentiation model in this study. The hMSC line expresses cell surface antigens (CD34-,

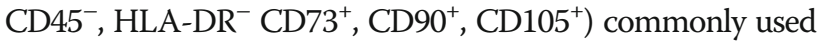
to characterize hMSC populations and can differentiate into mesodermal (osteogenic, adipogenic, chondrogenic) and even nonmesodermal (neural cells) lineages. The hMSC cell line was differentiated into sustainable neuronal morphology by combining several differentiation factors enriched with neurotrophic factors and NGF as well as BDNF [30, 34, 35] and differentiated cells express features specific to mature neural cells, such as Map 2, NF, and Tau. Thus, this immortalized cell line could be an important scientific tool to understand the in-vitro neural differentiation and degeneration mechanisms with a resemblance to the invivo situation.

Initially, morphological changes were observed in topo II $\beta$ silenced cells compared with untransfected cells. Topo II $\beta$ silenced hMSCs lost their fibroblastic morphology, becoming enlarged and flattened, and continued this morphology at further passages. It is known that topo II $\beta$ expression level and activity decreases during aging [8]. On the other hand, increasing donor age accelerates changes in hMSC morphology, and cells become enlarged in the culture even at early passages [31]. The morphology of hMSCs in the absence of topo II $\beta$ resembles the morphology of cells obtained from aged donors in our previous study [31]. Moreover, silencing of topo II $\beta$ expression before the day of neural induction prevented more than half of the cells from committing to neural differentiation and caused a dramatic decrease in neurite length, confirming previous studies $[3,10,11]$.

Attempts were made to understand the mechanism of neural differentiation in the absence of topo II $\beta$. The most well-established Rho-GTPases (RhoA, Cdc42, and Rac1) regulate axonogenesis in neuronal cells [36]. RhoA has been reported to negatively regulate neurite outgrowth during neural development, and its major downstream effector, Rock2, mediates RhoA-driven neurite retraction [17]. In our study, RhoA and Rock2 gene expressions were upregulated in response to topo II $\beta$ silencing. As Rock2 causes growth cone collapse and neurite retraction, these results suggest that upregulated RhoA and Rock2 gene activity may cause axon growth inhibition in the absence of topo II $\beta$. Furthermore, it is known that RhoA and Cdc42 can be antagonistic to one another in cells, and a balance in Rho-GTPase activities is required for the regulation of neurite outgrowth [36, 37]. In our study, Cdc42 was shown to be downregulated in topo II $\beta$-silenced cells, confirming the antagonistic effects of RhoA and Cdc42 in neurite outgrowth. On the other hand, a study by Chen et al. has shown that, during development of mice vertebrate central nervous system (CNS), deletion of Rac1 prevented axonal migration but not axonal outgrowth. In this study, no significant change was observed in the expression of Rac1 in topo II $\beta$ knockdown cells, supporting the hypothesis that Rac1 primarily controls axon guidance rather than outgrowth [38].

Axon growth inhibition, a degenerated neuronal network, and cell death following loss of cellular functions are common characteristics of several neurodegenerative diseases. 
Involvement of the RhoA/Rock2 signaling pathway has been suggested in neurodegenerative disorders such as AD, PD, HD, and ALS. Some nonsteroidal anti-inflammatory drugs (NSAIDs) have been shown to reduce the risk of neurodegeneration by blocking the RhoA/Rock 2 signaling pathway [17]. Inhibition of the RhoA/Rock2 pathway can be a therapeutic lead for diverse neurodegenerative disorders. Despite many treatment strategies for neurodegeneration, no full cure has been achieved because of the limited neurogenesis in the nervous system. Stem cell-based gene therapy shows potential as a powerful means of treating neurodegenerative disorders. Among adult stem cells, MSCs are one of the most extensively studied cell types with respect to their neural differentiation potential, and provide hope of developing therapeutics for neurodegenerative diseases [39]. There is ongoing research for MSCs as a therapeutic tool for AD, PD, and HD [21].

For this reason, we investigated the possible neuroprotective effect of topo II $\beta$ overexpression on the neural differentiated hMSC line to elucidate the further involvement of Rho-GTPases in topo II $\beta$-mediated axon growth. Topo II $\beta$ overexpression prompted all the cells to exhibit neural cell morphology as characterized by longer neurites. Our results indicate that topo $\mathrm{II} \beta$ is necessary for the commitment of the hMSC line to neural differentiation and promoting axon outgrowth.

Previous reports have indicated that RhoA/Rock2 inhibition can induce axonal regeneration after injury [40-44]. Thus, RhoA and Rock2 gene expression levels were analyzed in response to topo II $\beta$ overexpression. Our results demonstrated that overexpression of topo II $\beta$ causes a decrease in RhoA and Rock2 expression. Our findings suggest that topo II $\beta$ induces neurite outgrowth through inhibition of the RhoA/Rock2 pathway. On the other hand, Cdc42 was shown to be upregulated in topo II $\beta$ overexpressing cells, confirming a leading role in neurite outgrowth [36]. Another possible explanation for this is the opposing functions of RhoA and Cdc42, probably balanced by Rho regulator proteins. In contrast, no significant change was observed in the expression of Rac1, supporting the hypothesis that Rac1 may be responsible for other neurogenesis steps rather than axon growth in the neural differentiation process of hMSCs.

Studies performed in neural cells have found that Rac1 and Cdc42 activities are localized to the tips of the growing neurites [36]. In agreement with these studies, we also detected Rac1 and Cdc42 signals in the growing ends of axons in response to topo II $\beta$ overexpression, confirming the topo II $\beta$-dependent role of these GTPases in axon elongation. On the other hand, RhoA is localized to the cytoplasm and a low amount of endogenous RhoA is detected in the nuclear fraction [41]. In topo II $\beta$-overexpressing cells, RhoA signals were enriched in the nucleolus, whereas RhoA was not detectable in the nucleus and was restricted to the cytoplasm in topo II $\beta$-deficient cells. These data indicate that topo II $\beta$ causes a translocation of RhoA from the cytoplasm to the nucleus. It has been shown that nuclear localization of topo II $\beta$ is highly correlated with its catalytic activity. Active topo II $\beta$ is largely nucleoplasmic, while the nucleolar form is inactive [42]. It is possible that there could be a link between the subcellular localization of RhoA and its activity. In contrast to Dubash et al. [41], who found RhoA can be detected in the nucleus in its GTPbound active form, our results propose that inactive RhoA could accumulate in the nucleolus where it has limited access to the plasma membrane in topo II $\beta$ overexpressed neural cells. Furthermore, in response to topo II $\beta$ deficiency, RhoA could leave the nucleolus and target the cytoplasmic membrane upon activation.

Our findings suggest that Rho-GTPases can be a downstream target of topo II $\beta$; however, knowledge on the upstream regulation of topo II $\beta$ is also inadequate. Nurr1, a transcription factor belonging to the steroid/thyroid hormone receptor family, regulates the expression of target genes by binding to DNA. A study with Nurr1 knockout mice revealed that topo II $\beta$ is a downstream protein of Nurr1 and that Nurr1 regulates neurite outgrowth by regulating topo II $\beta$ gene expression [13]. The same study also showed that overexpression of topo II $\beta$ rescues Nurr1 deficiency-induced axon growth inhibition, indicating that topo II $\beta$ is the essential protein responsible for axon growth [13]. In our study, the expression pattern of Nurr1 in topo II $\beta$-silenced and -overexpressed hMSCs correlated with topo II $\beta$. One possible explanation for the regulation of Nurr1 gene expression according to topo II $\beta$ in the neural-induced hMSCs line is that the mechanism of topo II $\beta$ expression forms part of a feedback loop to regulate Nurr1 expression.

\section{Conclusions}

Based on the results of this study, it appears that inhibition of the RhoA/Rock 2 pathway by topo II $\beta$ overexpression can be used as a strategy to enhance the neural differentiation potential of MSCs and induce neurite outgrowth. A further study exploring the effect of topo II $\beta$-transfected primary MSC implantation on neurodegenerative disease animal models will provide us with a better understanding regarding the neuroprotector role of topo II $\beta$ in neurodegeneration.

\section{Additional files}

Additional file 1: Figure S1. Surface marker expressions of the hMSC line cells by flow cytometry. hMSC line cells were positive for CD90 (99.7\%), CD105 (99.7\%), and CD73 (99.8\%), and negative for CD45 (0.1\%), CD34 (0.5\%), and HLA-DR (0.1\%). (TIFF $553 \mathrm{~kb}$ )

Additional file 2: Figure S2. Multilineage mesodermal differentiation of the hMSC line cells. Undifferentiated (control) hMSCs (A), adipogenic 
differentiation stained with Oil Red O (B), osteogenic differentiation stained with Toluidine Blue $(C)$, and chondrogenic differentiation stained with Alcian Blue solution (D). Images X10. (TIFF $6680 \mathrm{~kb}$ )

\section{Abbreviations}

AD: Alzheimer's disease; ALS: Amyotrophic lateral sclerosis; BM-hMSC: Bone marrow-derived human mesenchymal stem cell; CGN: Cerebellar granule neuron; CN: Cortical neuron; dbCAMP: Dibutyryl cyclic AMP; DMEMLG: Dulbecco's modified Eagle's medium low glucose; DRG: Dorsal root ganglion; EGFP: Enhanced green fluorescent protein; FBS: Fetal bovine serum; FGF: Fibroblast growth factor; HD: Huntington's disease; hEGF: Human epidermal growth factor; hMSC: Human mesenchymal stem cell; IBMX: 3-Isobutyl-1-methylxanthine; MAP2: Microtubule-associated protein; mN3: Modified N3 medium; NF: Neurofilament; NGF: Nerve growth factor; NGS: Normal goat serum; NHS: Normal horse serum; NSAID: Nonsteroidal anti-inflammatory drug; p: Passage; PBS: Phosphate-buffered saline; PD: Parkinson's disease; rhBDNF: Recombinant human brain-derived neurotrophic factor; rhFGF: Recombinant human fibroblast growth factor; RT-qPCR: Reverse transcription quantitative polymerase chain reaction; SD: Standard deviation; topo II: Topoisomerase II

\section{Acknowledgements}

We sincerely thank Prof. Ken Tsutsui from the Okamaya University Medical School in Japan for donating GFP-topo $\| \beta$ plasmid. We also thank Derya Sultan Karabulut for technical support in the experiments.

\section{Funding}

This work was supported by a grant from the Scientific and Technological Research Council of Turkey (TUBITAK, grant no. 112S295).

\section{Availability of data and materials}

All data generated and/or analyzed during this study are included in this published article.

\section{Authors' contributions}

$\mathrm{MZ}$ and SI designed the research, analyzed the data, wrote the manuscript, and approved the final version.

\section{Ethics approval and consent to participate}

Not applicable.

\section{Competing interests}

The authors declare that they have no competing interests.

\section{Publisher's Note}

Springer Nature remains neutral with regard to jurisdictional claims in published maps and institutional affiliations.

\section{Received: 25 January 2018 Revised: 29 March 2018}

Accepted: 3 April 2018 Published online: 25 April 2018

\section{References}

1. Kawano S, et al. Regulation of DNA topoisomerase llbeta through RNAdependent association with heterogeneous nuclear ribonucleoprotein $\mathrm{U}$ (hnRNP U). J Biol Chem. 2010;285(34):26451-60.

2. McKinnon PJ. Topoisomerases and the regulation of neural function. Nat Rev Neurosci. 2016;17(11):673-9.

3. Tsutsui $\mathrm{K}$, et al. Involvement of DNA topoisomerase Illbeta in neuronal differentiation. J Biol Chem. 2001;276(8):5769-78.

4. Heng $X$, Le WD. The function of DNA topoisomerase llbeta in neuronal development. Neurosci Bull. 2010;26(5):411-6.

5. Yang $X$, et al. DNA topoisomerase Ilbeta and neural development. Science. 2000;287(5450):131-4.

6. Lyu YL, Wang JC. Aberrant lamination in the cerebral cortex of mouse embryos lacking DNA topoisomerase Ilbeta. Proc Natl Acad Sci U S A. 2003;100(12):7123-8.

7. Mandraju R, et al. Topoisomerase llbeta associates with Ku70 and PARP-1 during double strand break repair of DNA in neurons. Arch Biochem Biophys. 2011;516(2):128-37.
8. Kondapi AK, et al. Analysis of age dependent changes of topoisomerase II alpha and beta in rat brain. Int J Dev Neurosci. 2004;22(1):19-30.

9. Harkin $L F$, et al. Distinct expression patterns for type II topoisomerases IIA and IIB in the early foetal human telencephalon. J Anat. 2016;228(3):452-63.

10. Nur-E-Kamala A, Meiners S, Ahmed I, Azarova A, Lin CP, Lyu YL, Liu LF. Role of DNA topoisomerase $\| \beta$ in neurite outgrowth. Brain Res. 2007;1154:50-60.

11. Isik S, et al. DNA topoisomerase Illbeta as a molecular switch in neural differentiation of mesenchymal stem cells. Ann Hematol. 2015;94(2):307-18.

12. Nevin $L M$, et al. Topoisomerase llbeta is required for lamina-specific targeting of retinal ganglion cell axons and dendrites. Development. 2011;138(12):2457-65

13. Xin Heng GJ, Zhang X, Yang D, Zhu M, Shijun F, Li X, Le W. Nurr1 regulates Top Ilb and functions in axon genesis of mesencephalic dopaminergic neurons. Mol Neurodegener. 2012;7:4.

14. Takano T, Xu C, Funahashi Y, Namba T, Kaibuchi K. Neuronal polarization. Development. 2015;142(12):2088-93.

15. Hall A, Lalli G. Rho and Ras GTPases in axon growth, guidance, and branching. Cold Spring Harb Perspect Biol. 2010;2(2):a001818.

16. Yamamoto K, et al. The novel Rho kinase (ROCK) inhibitor K-115: a new candidate drug for neuroprotective treatment in glaucoma. Invest Ophthalmol Vis Sci. 2014;55(11):7126-36.

17. Fujita Y, Yamashita T. Axon growth inhibition by RhoA/ROCK in the central nervous system. Front Neurosci. 2014:8:338.

18. Linseman DA, Loucks FA. Diverse roles of Rho family GTPases in neuronal development, survival, and death. Front Biosci. 2008;13:657-76.

19. Stankiewicz TR, Linseman DA. Rho family GTPases: key players in neuronal development, neuronal survival, and neurodegeneration. Front Cell Neurosci. 2014;8:314.

20. Taupin P. Adult neural stem cells, neurogenic niches, and cellular therapy. Stem Cell Rev. 2006;2(3):213-9.

21. Gu H, et al. Inhibition of the Rho signaling pathway improves neurite outgrowth and neuronal differentiation of mouse neural stem cells. Int J Physiol Pathophysiol Pharmacol. 2013;5(1):11-20

22. Ming GL, Song H. Adult neurogenesis in the mammalian brain: significant answers and significant questions. Neuron. 2011;70(4):687-702.

23. Takeda YS, Xu Q. Neuronal differentiation of human mesenchymal stem cells using exosomes derived from differentiating neuronal cells. PLoS One. 2015:10(8):e0135111.

24. Akazawa $Y$, et al. Recruitment of mesenchymal stem cells by stromal cellderived factor 1alpha in pulp cells from deciduous teeth. Int J Mol Med. 2015;36(2):442-8.

25. Hasegawa T, et al. Differential effects of TGF-beta1 and FGF-2 on SDFlalpha expression in human periodontal ligament cells derived from deciduous teeth in vitro. Int J Mol Med. 2012:30(1):35-40.

26. Mori T, et al. Combination of hTERT and bmi-1, E6, or E7 induces prolongation of the life span of bone marrow stromal cells from an elderly donor without affecting their neurogenic potential. Mol Cell Biol. 2005;25(12):5183-95.

27. Shimomura $\mathrm{T}$, et al. Hepatic differentiation of human bone marrow-derived UE7T-13 cells: effects of cytokines and CCN family gene expression. Hepatol Res. 2007;37(12):1068-79.

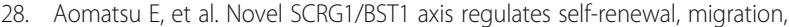
and osteogenic differentiation potential in mesenchymal stem cells. Sci Rep. 2014:4:3652.

29. Pfaffl MW. A new mathematical model for relative quantification in real-time RT-PCR. Nucleic Acids Res. 2001;29(9):e45.

30. Long $X$, et al. Neural cell differentiation in vitro from adult human bone marrow mesenchymal stem cells. Stem Cells Dev. 2005;14(1):65-9.

31. Zaim $M$, et al. Donor age and long-term culture affect differentiation and proliferation of human bone marrow mesenchymal stem cells. Ann Hematol. 2012;91(8):1175-86.

32. Wagner W, et al. Replicative senescence of mesenchymal stem cells: a continuous and organized process. PLoS One. 2008;3(5):e2213.

33. Sethe S, Scutt A, Stolzing A. Aging of mesenchymal stem cells. Aging Res Rev. 2006:5(1):91-116.

34. Caldwell MA, et al. Growth factors regulate the survival and fate of cells derived from human neurospheres. Nat Biotechnol. 2001;19(5):475-9.

35. Johansson PA, Cappello S, Gotz M. Stem cells niches during development-lessons from the cerebral cortex. Curr Opin Neurobiol. 2010;20(4):400-7.

36. Koh CG. Rho GTPases and their regulators in neuronal functions and development. Neurosignals. 2006;15(5):228-37. 
37. Woo S, Gomez TM. Racl and RhoA promote neurite outgrowth through formation and stabilization of growth cone point contacts. J Neurosci. 2006;26(5):1418-28.

38. Chen $L$, et al. Rac1 controls the formation of midline commissures and the competency of tangential migration in ventral telencephalic neurons. $J$ Neurosci. 2007;27(14):3884-93.

39. Taran R, et al. In vitro and in vivo neurogenic potential of mesenchymal stem cells isolated from different sources. J Biosci. 2014;39(1):157-69.

40. Yuan XB, et al. Signalling and crosstalk of Rho GTPases in mediating axon guidance. Nat Cell Biol. 2003;5(1):38-45.

41. Dubash AD, et al. The small GTPase RhoA localizes to the nucleus and is activated by Net1 and DNA damage signals. PLoS One. 2011;6(2):e17380.

42. Onoda A, Hosoya O, Sano K, Kiyama K, Kimura H, Furuta R, Kawano S, Miyaji M, Tsutsui K, Tsutsui KM. Nuclear dynamics of topoisomerase $\| \beta$ reflects its catalytic activity that is regulated by binding of RNA to the C-terminal domain. Nucleic Acids Res. 2014;42(14):9005-20.

43. Schmandke A, Schmandke A, Strittmatter SM. ROCK and Rho: biochemistry and neuronal functions of Rho-associated protein kinases. Neuroscientist. 2007;13(5):454-69.

44. Fournier AE, Takizawa BT, Strittmatter SM. Rho kinase inhibition enhances axonal regeneration in the injured CNS. J Neurosci. 2003;23(4):1416-23.

Ready to submit your research? Choose BMC and benefit from:

- fast, convenient online submission

- thorough peer review by experienced researchers in your field

- rapid publication on acceptance

- support for research data, including large and complex data types

- gold Open Access which fosters wider collaboration and increased citations

- maximum visibility for your research: over $100 \mathrm{M}$ website views per year

At BMC, research is always in progress.

Learn more biomedcentral.com/submissions 